\title{
Penetration of Commercial and Dental Waxes
}

\author{
J. M. POWERS and R. G. CRAIG
}

School of Dentistry, University of Michigan, Ann Arbor, Michigan 48104, USA

Penetration of commercial and dental waxes was studied. Measurements indicated that resistance of paraffin and dental inlay waxes to penetration was closely related to the tem. perature at which solid-solid transformations occurred. Annealed waxes were more resistant to penetration than unannealed waxes.

Thermomechanical analysis (TMA) measures the mechanical response of a material as it is programmed linearly through a selected temperature range. 1 The techniques used in TMA include linear expansion, volumetric expansion, tensile elongation, torsional modulus, and penetration. Comparison of TMA penetration curves with curves of transition temperatures obtained from differential thermal analysis (DTA), is a powerful, new technique for studying the effect of thermal transitions of a material on its mechanical properties.

DTA of commercial and dental waxes has established the presence of solid-solid transition temperatures as well as melting transitions. ${ }^{2}$ The phase changes associated with these transition temperatures might be expected to influence the mechanical behavior of wax. In this study we examine the relationship between penetration and transition temperatures for various commercial, dental, and combined waxes and provide fundamental information on the formulation and testing of dental waxes.

\section{Materials and Methods}

Waxes, as obtained from the supplier (Table 1) or blended as indicated, were subjected to penetration. Cylindrical wafers (6.0 $\mathrm{mm}$ in diameter and $1.0 \mathrm{~mm}$ in height) of a wax were made in a warmed stainless

This paper was presented, in part, dt the 51 st general session of the IADR in Washington, DC.

Received for publication March 26, 1973. steel die. The temperature of the die was the annealing temperature for a particular wax or wax blend.

The waxes were studied in annealed and unannealed conditions to test the effect of heat treatment on penetration. Annealed specimens were prepared in a manner similar to the unannealed specimens, but were heattreated in an oven for 24 hours at a temperature $20 \mathrm{C}$ below the melting temperature of the wax before testing. The temperatures at which the various waxes were annealed are given in Table 2.

TMA $^{\mathrm{a}}$ was carried out on three specimens for each condition from $25 \mathrm{C}$ to the temperature at which maximum $(100 \%)$ penetration occurred for the various waxes. The analysis was conducted in air, and a linear heating rate of $5 \mathrm{C}$ per minute was used. DTA ${ }^{b}$ was carried out from 25 to $100 \mathrm{C}$ in air with a heating rate of $20 \mathrm{C}$ per minute.

A schematic sketch of the penetration mode of the thermomechanical analysis cell is shown in Figure 1. A quartz probe, which is attached to a metallic sleeve that serves at the core of a linear variable differential transformer (LVDT), was mechanically positioned by a spring on the surface of a wax sample. The output of the LVDT was then electrically balanced and zeroed so subsequent vertical displacement could be calibrated and recorded as a function of temperature. The cylindrical probe tip shown in the blown-up portion of Figure 1 has a diameter of $0.92 \mathrm{~mm}$ and a length of 0.72 $\mathrm{mm}$. Thus, $100 \%$ penetration refers to a vertical displacement of $0.72 \mathrm{~mm}$. Stresses of 1.52 and $25.5 \mathrm{gm} / \mathrm{mm}^{2}$ were applied to the wax. All TMA curves began at $25 \mathrm{C}$ with

a DuPont 941 Thermomechanical Analyzer, E. I. duPont de Nemours \& Co., Inc, Instrument Products Division, Wilmington, Del.

b DuPont 900 Differential Thermal Analyzer, E. I. duPont de Nemours \& Co., Inc, Instrument Products Division, Wilmington, Del. 
TABLE 1

Commercial, Dental, and Combined Waxes

\begin{tabular}{lll}
\hline \hline \multicolumn{1}{c}{ Wax } & \multicolumn{1}{c}{ Description } \\
\hline Paraffin & Fully refined paraffin wax (lot 319) & Rource \\
Beeswax & Refined yellow beeswax, USP (lot 2247) & Ross Co., Jersey City, NJ \\
Carnauba & Refined pure no. l yellow carnauba (lot 2318) & Ross Co., Jersey City, NJ \\
$D D$ & Blue inlay casting wax, regular (lot 0141 B806) & Kerr Mfg. Co., Romulus, Mich \\
$E E$ & Blue inlay casting wax, hard (lot 0824A865) & Kerr Mfg. Co., Romulus, Mich \\
$F F$ & Dr. Peck's purple hard wax (lot 1136) & Kerr Mfg. Co., Romulus, Mich \\
$J$ & Blue inlay wax, regular (lot 0727101) & S. S. White Co., Philadelphia, Pa \\
$K$ & Blue inlay wax, hard (lot 20912) & S. S. White Co., Philadelphia, Pa \\
\hline
\end{tabular}

zero percent penetration. When several curves were plotted on the same figure, the curves were offset for convenience. Temperatures at which 10,50, and $90 \%$ penetration occurred were analyzed statistically by analysis of variance. ${ }^{3}$ A hypothesis testing the equivalence of means was rejected if the computed value of $\alpha$ was less then or equal to 0.05

\section{Results}

The relationship between DTA and TMA by penetration for annealed carnauba wax is shown in Figure 2. The TMA curves for both stress levels reached about $80 \mathrm{C}$ before penetration was observed. Initiation of penetration at both stress levels was associated with the endothermic DTA transition ob-

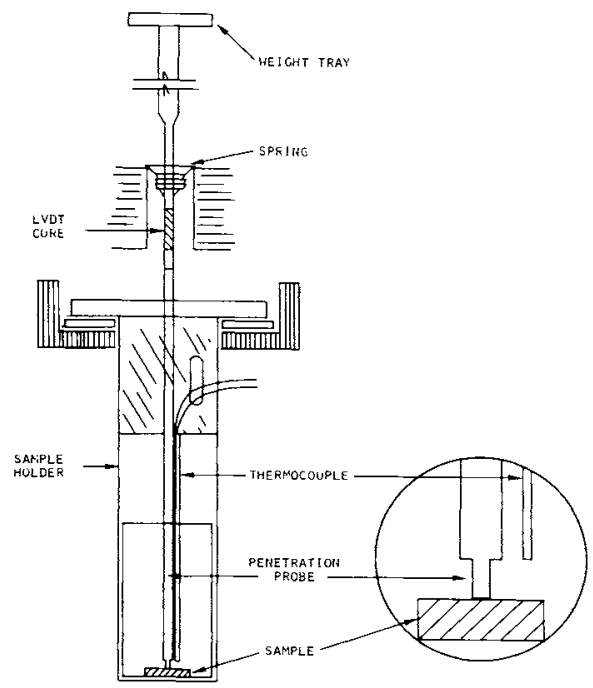

FIG 1.-Schematic sketch of penetration mode of TMA cell.
TABLE 2

Annealing Temperatures for Commercial, Dental, and Combined WaXes

\begin{tabular}{lc}
\hline \multicolumn{1}{c}{ Wax } & $\begin{array}{c}\text { Annealing } \\
\text { Temperature }\end{array}$ \\
\hline Paraffin & $40 \mathrm{C}$ \\
Paraffin-beeswax blends & $40 \mathrm{C}$ \\
Beeswax & $40 \mathrm{C}$ \\
Paraffin-carnauba blends & \\
$\quad(2.5$ to $25 \%$ carnauba) & $40 \mathrm{C}$ \\
Paraffin-carnauba blends & \\
$\quad(50$ to $75 \%$ carnauba) & $60 \mathrm{C}$ \\
Carnauba & $60 \mathrm{C}$ \\
$D D$ & $50 \mathrm{C}$ \\
$E E$ & $50 \mathrm{C}$ \\
$F F$ & $50 \mathrm{C}$ \\
$J$ & $50 \mathrm{C}$ \\
$K$ & $50 \mathrm{C}$ \\
\hline
\end{tabular}

served from 65 to $90 \mathrm{C}$. Mean temperatures at which penetration of 10,50 , and $90 \%$ occurred for carnauba was at both stress levels are listed in Table 3 .

The relationship between DTA and TMA for annealed beeswax is shown in Figure 3 . The curve for the lower stress level was associated with the endothermic transition ob-

TABLE 3

Mean Penetration Temperatures for Carnauba and BeEswax at Two Stress LeVels

\begin{tabular}{crcc}
\hline \hline Stress & Penetration & Carnauba & Beeswax \\
\hline $1.52 \mathrm{gm} / \mathrm{mm}^{2}$ & $10 \%$ & $84.0(1.8) *$ & $57.1(6.3)$ \\
& $50 \%$ & $85.7(1.8)$ & $61.3(0.8)$ \\
& $90 \%$ & $86.5(1.9)$ & $61.7(0.8)$ \\
$25.5 \mathrm{gm} / \mathrm{mm}^{2}$ & $10 \%$ & $81.0(0.3)$ & $37.8(0.6)$ \\
& $50 \%$ & $82.1(0.4)$ & $44.0(0.4)$ \\
& $90 \%$ & $82.1(0.3)$ & $44.6(0.4)$ \\
\hline
\end{tabular}

Note: All means were significantly different at the $95 \%$ level when compared between stress levels.

- Mean (standard deviation) with sample size of three; units in degrees $C$. 


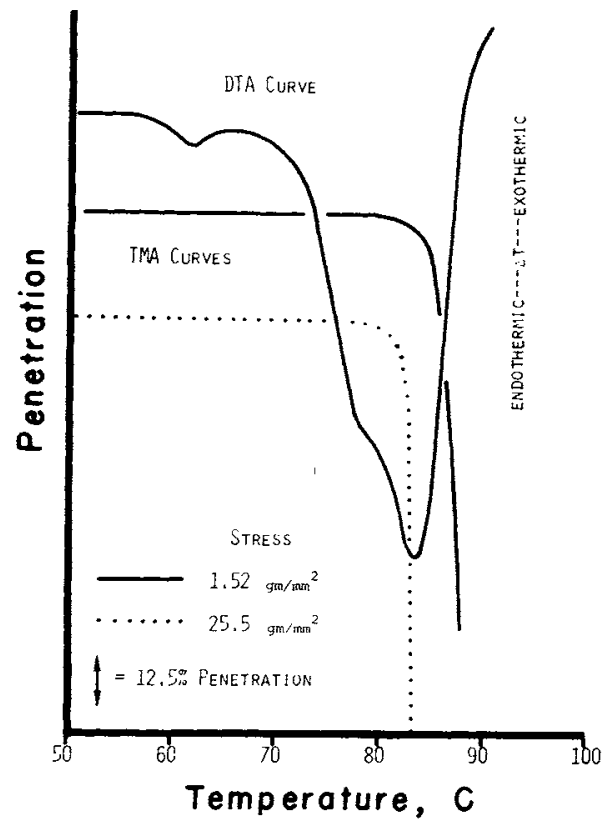

Frc 2.-Relationship between DTA and TMA by penetration for carnauba wax at two stress levels.

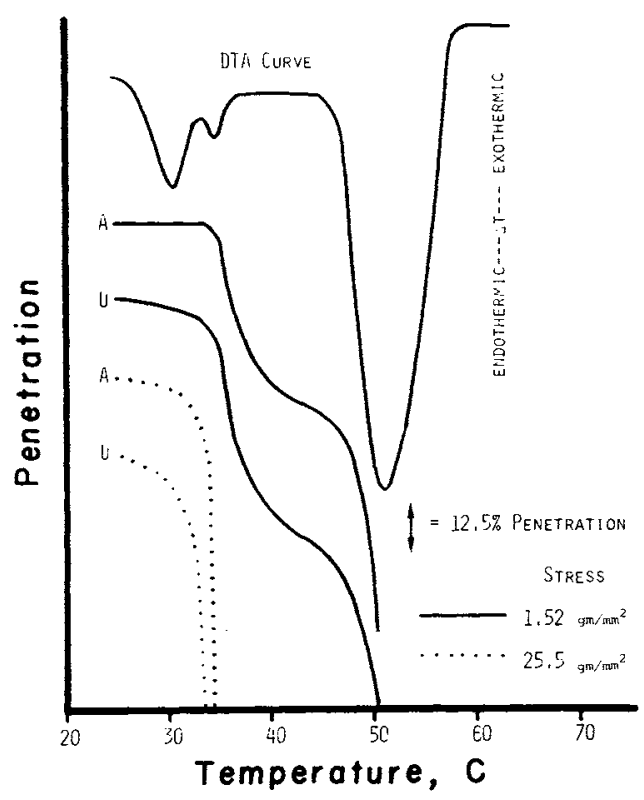

Fig 4.-Relationship between DTA and TMA by penetration for annealed $(A)$ and unannealed (U) paraffin at two stress levels.

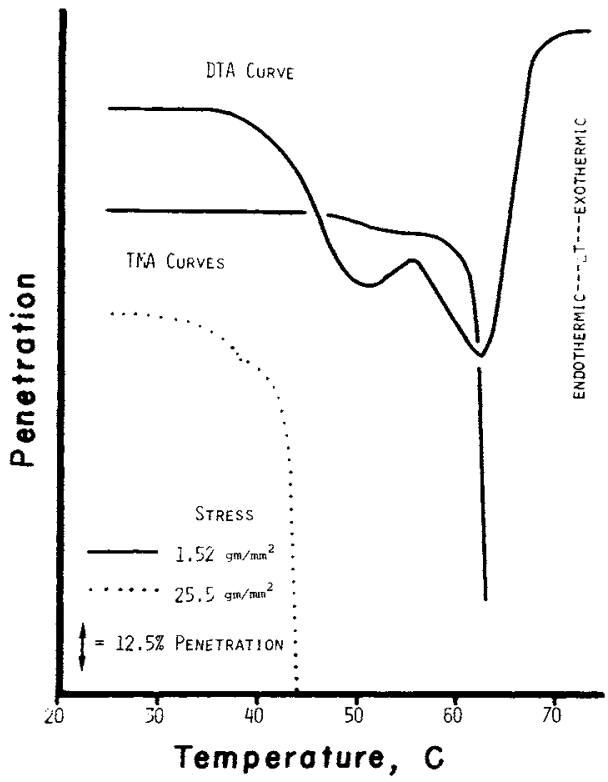

Fic 3.-Relationship between DTA and TMA by penetration for beeswax at two stress levels.

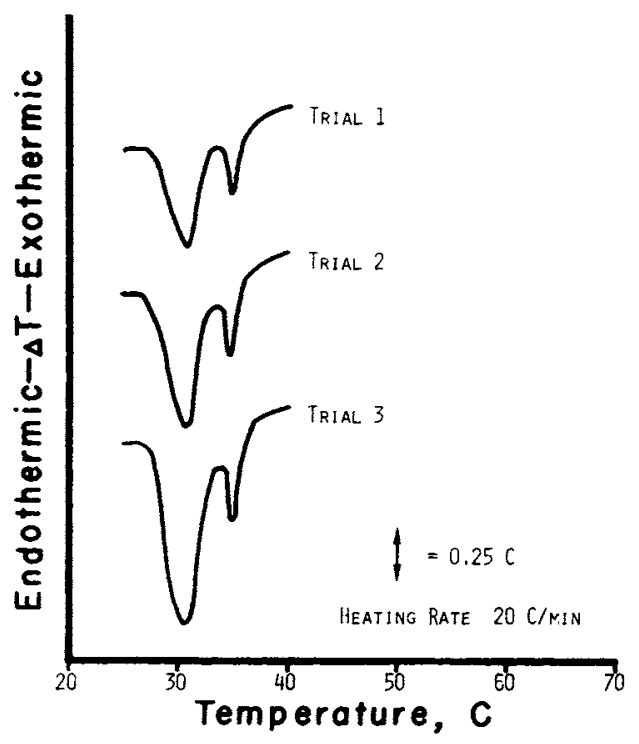

Fig 5.-Influence of annealing on $\Delta T$ of premelting transitions of paraffin. 
TABLE 4

Mean Penetration Temperatures for Annealed and Unannealed Paraffin at T'wo Stress Levels

\begin{tabular}{clccc}
\hline & & \multicolumn{3}{c}{ Penetration } \\
\cline { 3 - 5 } Stress & Treatment & $10 \%$ & $50 \%$ & $90 \%$ \\
\hline $1.52 \mathrm{gm} / \mathrm{mm}^{2}$ & Unannealed & $34.2(0.2) *$ & $38.4(0.9)$ & $49.1(0.3)$ \\
& Annealed & $35.0(0.5)$ & $46.8(0.3)$ & $50.0(0.2)$ \\
$25.5 \mathrm{gm} / \mathrm{mm}^{2}$ & Unannealed & $29.1(1.7)$ & $33.8(0.3)$ & $34.4(0.4)$ \\
& Annealed & $33.1(0.3)$ & $34.2(0.5)$ & $34.4(0.5)$ \\
\hline
\end{tabular}

Note: All means were significantly different at the $95 \%$ level when compared between stress levels and when compared between annealed and unannealed conditions at the lower stress level. At the higher stress level, there was a significant difference at the $95 \%$ level between means of annealed and unannealed samples at $10 \%$ penetration only.

- Mean (standard deviation) with sample size of three; units in degrees $C$.

served from 55 to $70 \mathrm{C}$. An average initial penetration of about $15 \%$ was observed at $52 \mathrm{C}$ at this stress level. The curve for the higher stress level was associated with the onset of the endothermic transition observed from 53 to $55 \mathrm{C}$.

The relationship between DTA and TMA for annealed and unannealed paraffin shown in Figure 4. The curve for annealed paraffin at the lower stress level had an initial penetration associated with the initial endothermic transitions observed for paraffin from 25 to $38 \mathrm{C}$. This initial penetration accounted for about $45 \%$ of the total penetration. The final penetration was associated with the

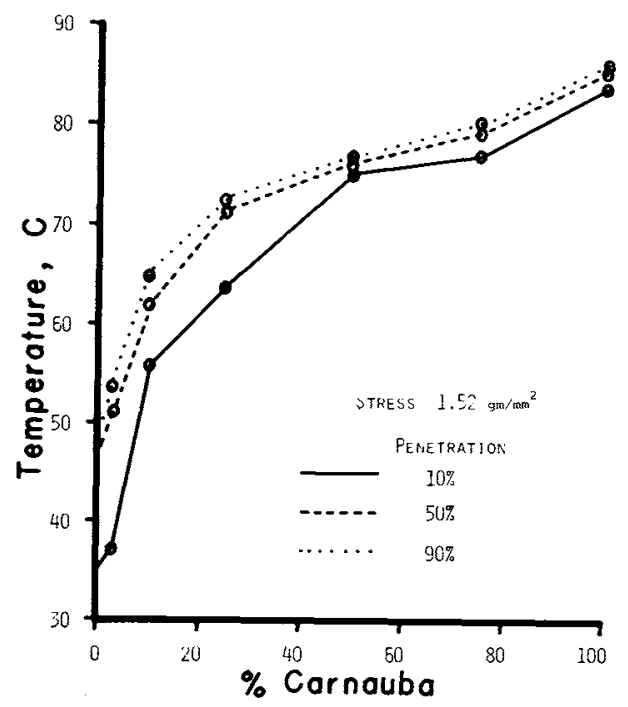

Fig 6.-Penetration temperature vs percentage carnauba for paraffin-carnauba binary mixtures at a stress level of $1.52 \mathrm{gm} / \mathrm{mm}^{2}$. large endothermic transition observed from 45 to $58 \mathrm{C}$. The curve for annealed paraffin at the higher stress level was associated entirely with the initial endothermic transitions of paraffin. The mean penetration temperatures for annealed and unannealed paraffin are shown in Table 4.

To explain the differences observed be. tween the annealed and unannealed conditions of paraffin, a limited study was made of the influence of annealing (heat treatment) on the magnitude of the endothermic transitions observed from 25 to $38 \mathrm{C}$ for paraffin. The results of this DTA study are shown in Figure 5. Trial $l$ is the DTA curve of an unannealed sample of paraffin run from 25 to $40 \mathrm{C}$. Trial 2 is the DTA curve of the sample of paraffin that was tested immediately after cooling from trial 1. Trial 3 is the DTA curve of the sample of paraffin from trial 2 that was annealed at $40 \mathrm{C}$ for 24 hours before testing. The differential temperatures or peak heights $(\Delta T)$ observed for the endothermic transition with a maximum at $31 \mathrm{C}$ were $4.5,6.0$, and $8.5 \mathrm{C}$ for the three trials, respectively. The values of $\Delta \mathrm{T}$ observed for the endothermic transition with a maximum at $34.5 \mathrm{C}$ were $2.0,2.25$, and $2.5 \mathrm{C}$ respectively.

Mean temperatures for penetration of 10 , 50 , and $90 \%$ at a stress level of $1.52 \mathrm{gm} / \mathrm{mm}^{2}$ are shown in Figure 6 for annealed binary mixtures of paraffin and carnauba.

Mean temperatures for penetration of 10 , 50 , and $90 \%$ at a stress level of $1.52 \mathrm{gm} / \mathrm{mm}^{2}$ are shown in Figure 7 for annealed binary mixtures of paraffin and beeswax. Each circled data point is the mean of three replications.

The temperature data for $10 \%$ penetra- 


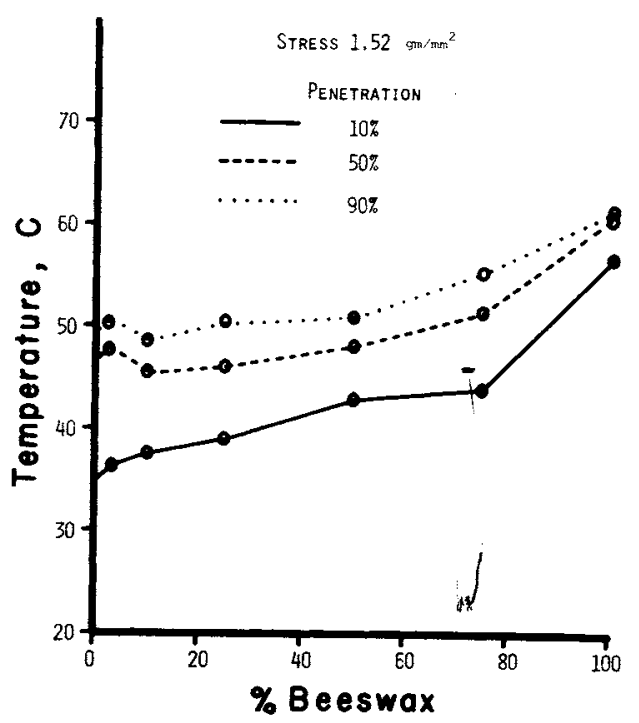

Fig 7.-Penetration temperature vs percentage beeswax for paraffin-beeswax binary mixtures at a stress level of $1.52 \mathrm{gm} / \mathrm{mm}^{2}$.

tion for both beeswax-paraffin and carnaubaparaffin binary mixtures at each stress level is summarized in Figure 8. The most noticeable features of these data are the great ef-

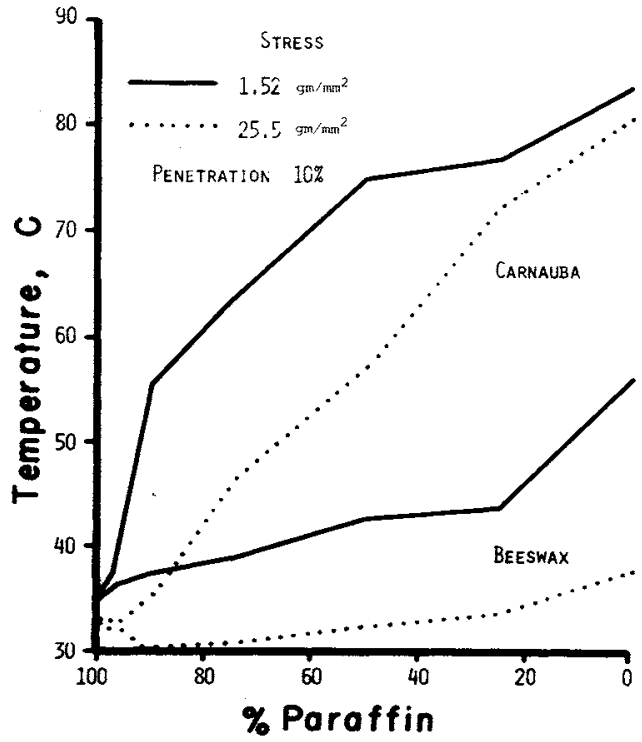

Fig 8.-Penetration temperature vs percentage paraffin for binary mixtures of paraffin with beeswax or carnauba for penetrations of $10 \%$ at two stress levels.

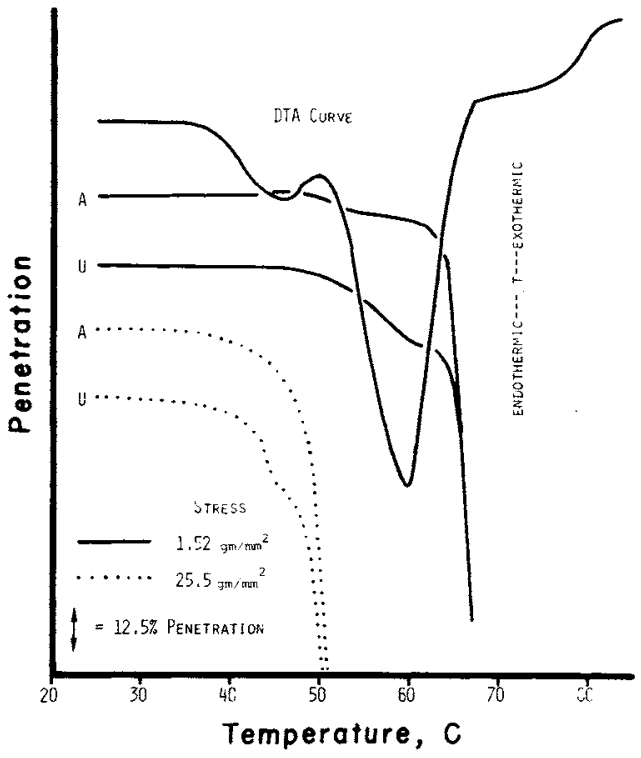

Fic 9.-Relationship between DTA and TMA by penetration for annealed $(A)$ and unannealed (U) dental inlay wax at two stress levels.

fect of small additions of carnauba wax and the almost negligible effect of small additions of beeswax on increasing the resistance to penetration of the binary mixtures. The effect of higher stress on $10 \%$ penetration temperatures of the pure components also is shown in Figure 8. The resistance to penetration of beeswax was influenced to a greater extent by increased stress then that of paraffin or carnauba. The differences in penetration temperatures at the two stress levels for $10 \%$ penetration observed for beeswax, carnauba, and paraffin were 19.3, 3.0, and $2.9 \mathrm{C}$, respectively.

The relationship between DTA and TMA for annealed and unannealed dental inlay wax is shown in Figure 9. The curve for the annealed wax at the lower stress level had an initial penetration that was associated with the onset of the major endothermic transition observed for the dental wax from 50 to $68 \mathrm{C}$. This initial penetration accounted for about $10 \%$ of the total penetration. The final penetration was associated with the completion of the major transition and the onset of the endothermic transition observed from 68 to $85 \mathrm{C}$. The curve for the annealed wax at the higher stress level was associated entirely with the endothermic transition ob- 
TABLE 5

Mean Penetration Temperatures for five Annealed Dental Inlay Waxes at Two Stress Levels

\begin{tabular}{ccccccc}
\hline \hline & & \multicolumn{5}{c}{ Waxes } \\
\cline { 3 - 7 } Stress & Penetration & DD & EE & $F F$ & $J$ & $K$ \\
\hline $1.52 \mathrm{gm} / \mathrm{mm}^{2}$ & $10 \%$ & $61.4(1.9) *$ & $63.4(1.1)$ & $65.2(1.0)$ & $61.1(0.9)$ & $63.9(0.6)$ \\
& $50 \%$ & $67.3(0.7)$ & $66.1(0.7)$ & $68.6(0.4)$ & $66.3(0.2)$ & $66.5(0.2)$ \\
& $90 \%$ & $68.1(0.8)$ & $66.9(0.6)$ & $69.1(0.3)$ & $67.4(0.2)$ & $67.3(0.2)$ \\
$25.5 \mathrm{gm} / \mathrm{mm}^{2}$ & $10 \%$ & $44.9(1.0)$ & $44.9(1.1)$ & $47.8(1.8)$ & $39.8(1.0)$ & $44.5(1.0)$ \\
& $50 \%$ & $49.1(0.3)$ & $50.5(0.6)$ & $54.7(1.0)$ & $46.3(0.4)$ & $49.0(0.2)$ \\
& $90 \%$ & $50.2(0.2)$ & $52.0(0.6)$ & $56.9(0.9)$ & $47.8(0.2)$ & $50.4(0.2)$ \\
\hline
\end{tabular}

- Mean (standard deviation) with sample size of three; units in degrees C.

served from 35 to $50 \mathrm{C}$. The data shown in Figure 9 are typical of the five dental inlay waxes studied.

Mean temperatures at which penetrations of 10,50 , and $90 \%$ occurred for five annealed dental inlay waxes at both stress levels are listed in Table 5. An analysis of variance of penetration temperatures of annealed waxes (Table 5) indicated significant differences among means at levels of $\alpha$ of less than 0.05 . Ranking of the means is shown in Table 6 . In general, Type I waxes were more resistant to penetration than Type II waxes at the higher stress level.

The temperature data for $10 \%$ penetration for dental inlay waxes at each stress level is summarized in Table 7 for the annealed and unannealed conditions. In general, annealed dental inlay waxes were more resistant to penetration, particularly at the higher stress level. This observation is further demonstrated by examination of the penetration data (percentages) at temperatures of 30 and $37 \mathrm{C}$ listed in Table 8 for annealed and

TABLE 6

Ranking of Mean Penetration Temperatures of ANNEAled Dental Inlay Waxes at Two STress Levels

\begin{tabular}{|c|c|c|c|c|c|c|}
\hline Stress & Penetratio & & & Waxes & & \\
\hline \multirow[t]{3}{*}{$1.52 \mathrm{gm} / \mathrm{mm}^{2}$} & $10 \%$ & $J$ & $D D$ & $E E$ & $K$ & $F F$ \\
\hline & $50 \%$ & $E E$ & $J$ & $K$ & $D D$ & $F F$ \\
\hline & $90 \%$ & $E E$ & $K$ & $J$ & $D D$ & $F F$ \\
\hline \multirow[t]{3}{*}{$25.5 \mathrm{gm} / \mathrm{mm}^{2}$} & $10 \%$ & $J$ & $K$ & $D D$ & $E E$ & $F F$ \\
\hline & $50 \%$ & $J$ & $K$ & $D D$ & $E E$ & $F F$ \\
\hline & $90 \%$ & $J$ & $D D$ & $K$ & $E E$ & $F F$ \\
\hline
\end{tabular}

Note: Underscoring indicates no significant difference $(a=0.05)$. unannealed conditions of the five dental inlay waxes.

\section{Discussion}

Comparison of the penetration curves observed for beeswax, carnauba, and paraffin with DTA measurements ${ }^{2}$ indicates that resistance to penetration is closely related to solid-solid and melting transitions. The extent to which penetration occurs appears to be dependent on the temperature at which the phase change occurs and the extent to which this transformation takes place. Ex. perimentally, penetration is dependent on factors such as time, temperature, load, and rate of heating. The rate of heating is especially critical for materials that are poor thermal conductors, such as waxes.

DTA measurements ${ }^{2}$ indicate that the beeswax used in this study is a mixture of hydrocarbon and ester waxes, each of which has a distinct endothermic melting transition. At the lower stress, the higher melting component provides sufficient integrity to the wax for it to resist penetration, although the lower melting component has undergone a major phase transition. The resistance of beeswax to penetration is influenced at high stress levels by the temperature at which the lower melting component transforms.

Carnauba wax is a relatively pure, highmelting ester wax. ${ }^{2}$ Penetration at both stress levels corresponds to the melting transformation: penetration at the higher stress level occurs at the onset of the melting transition and penetration at the lower stress level occurs near the completion of the melting transition. At the two levels of stress studied, the solid-solid transition of carnauba, which occurred at $61.0 \mathrm{C}$, had no influence on penetration. 
TABLE 7

Summary of Temperatures at 10\% Penetration for five Dental Inlay Waxes in Annealed and Unannealed Conditions at Two Stress Levels

\begin{tabular}{clccccc}
\hline \multirow{2}{*}{ Stress } & \multicolumn{5}{c}{ Waxes } \\
\cline { 3 - 7 } & Treatment & $D D$ & $E E$ & $F F$ & $J$ & $K$ \\
\hline $1.52 \mathrm{gm} / \mathrm{mm}^{2}$ & Unannealed & $56.8(7.0) *$ & $56.9(0.4)$ & $63.1(6.6)$ & $48.8(1.5)$ & $57.4(7.7)$ \\
& Annealed & $61.4(1.9)$ & $63.4(1.1)$ & $65.2(1.0)$ & $61.1(0.9)$ & $63.9(0.6)$ \\
$25.5 \mathrm{gm} / \mathrm{mm}^{2}$ & Unannealed & $46.5(1.0)$ & $43.0(0.1)$ & $44.1(1.8)$ & $37.4(0.8)$ & $43.1(1.8)$ \\
& Annealed & $44.9(1.0)$ & $44.9(1.1)$ & $47.8(1.8)$ & $39.8(1.0)$ & $44.5(1.0)$ \\
& & &
\end{tabular}

Note: All means were significantly different at the $95 \%$ level when compared between annealed and unannealed conditions.

- Mean (standard deviation) with sample size of three; units in degrees $\mathrm{C}$.

The paraffin used in this study is a lowmelting $(51 \mathrm{C})$, hydrocarbon wax that undergoes two solid-solid phase transformations about 16 to $20 \mathrm{C}$ below its melting transition. ${ }^{2}$ An initial penetration accompanied the lower temperature solid-solid transformation at the lower stress level. Final penetration occurred with the onset of the melting transition. At the higher stress level, penetration was associated entirely with the lower temperature solid-solid phase transformation. Thus, the resistance of paraffin to penetration is predominantly influenced by the temperature at which the solid-solid phase transformation occurs and, to a lesser extent, by the melting temperature.

Annealing paraffin at $40 \mathrm{C}$ for 24 hours was shown to increase the resistance to penetration. A corresponding increase in the magnitude of the heat of transition of primarily the lower temperature phase transformation was a result of this heat treatment. Annealing paraffin increases the crystallinity of the lattice primarily with respect to the oddnumbered paraffin hydrocarbons, 4,5 and this increased crystallinity offers more resistance to penetration.
The pronounced effects of small additions of carnauba on the increased resistance of penetration of paraffin are consistent with DTA measurements. ${ }^{2}$ Addition of beeswax to paraffin does not produce pronounced hardening; rather, it appears that small additions of beeswax may actually reduce the resistance of paraffin to penetration by interfering with the crystallinity of the paraffin.

DTA measurements ${ }^{2}$ suggest that dental inlay waxes are mixtures of hydrocarbon and ester waxes. The effect of the high-melting ester component on the resistance to penetration of dental inlay waxes at low stress levels is apparent. Although there is an initial penetration associated with the melting transformation of the hydrocarbon wax, the major penetration is beyond the melting transition of the hydrocarbon wax. At high stress levels, however, the temperature of the solid-solid transformation associated with the hydrocarbon is the determining factor of the resistance to penetration. With most of the dental inlay waxes studied, this solid-solid transition occurred well above the temperature of the mouth. ${ }^{2}$ Formulation of waxes where resistance to flow is important should

TABLE 8

Penetration Percentages for five Dental Inlay Waxes in Annealed and Unannealed Conditions at Two Temperatures at a Stress Level of $25.5 \mathrm{gm} / \mathrm{mm}^{2}$

\begin{tabular}{cllcccc}
\hline & & \multicolumn{5}{c}{ Waxes } \\
\cline { 3 - 7 } Temperature & Treatment & $D D$ & $E E$ & $F F$ & $J$ & $K$ \\
\hline \multirow{2}{*}{$30 \mathrm{C}$} & Unannealed & $0.9(0.4)^{*}$ & $0.5(0.0)$ & $0.9(0.4)$ & $1.5(0.2)$ & $0.8(0.6)$ \\
& Annealed & $0.1(0.1)$ & $0.0(0.1)$ & $0.4(0.3)$ & $0.7(0.2)$ & $0.7(0.6)$ \\
$37 \mathrm{C}$ & Unannealed & $2.4(1.4)$ & $2.2(0.1)$ & $5.0(0.1)$ & $9.6(1.7)$ & $4.2(1.0)$ \\
& Annealed & $1.4(0.2)$ & $0.8(0.2)$ & $1.3(1.1)$ & $4.7(1.3)$ & $2.6(2.0)$ \\
\hline
\end{tabular}

Note: All means were significantly different at the $95 \%$ level when compared between annealed and unannealed conditions.

- Mean (standard deviation) with sample size of three; units in percentages. 
be based more on the temperature at which the solid-solid transition occurs than on the melting temperature of the hydrocarbon wax. The importance of the solid-solid transition on the resistance to penetration of a wax blend, however, decreases greatly as increasing amounts of high-melting ester wax are added.

Annealing the dental inlay waxes at $50 \mathrm{C}$ for 24 hours improves their resistance to penetration and decreases the variance associated with the measurement. It was not determined if these effects were the results of an increased crystallinity of the hydrocarbon component.

Because the results show that resistance to penetration of dental waxes may be significantly changed by heat treatment, the current practice of storing a wax at $37 \mathrm{C}$ for 24 hours before testing for recommended properties may be misleading. Future studies should examine the effect of heat treatment on a wax to determine if critical factors exist.

\section{Conclusions}

Penetration curves from $25 \mathrm{C}$ have been determined by TMA for a variety of commercial, dental, and combined waxes. Comparison of these curves with DTA measurements indicated that resistance of waxes to penetration was closely related to the temperature at which solid-solid and melting transformations occurred.

The resistance of beeswax to penetration was influenced at high stress levels by the temperature at which its lower melting component transformed. At low stress levels, resistance to penetration was dependent on the transformation temperatures of both components. The resistance to penetration of carnauba was determined primarily by its melting transition.

The resistance to penetration of paraffin was predominantly influenced by the temperature at which the major solid-solid transformation occurred. A heat treatment that increased the heat of transition associated with the major solid-solid transition of paraffin also increased the resistance to penetration of the annealed paraffin when compared with the unannealed paraffin.

Additions of carnauba to paraffin caused a pronounced increase in the resistance of the mixture to penetration; addition of beeswax to paraffin did not produce comparable results.

At high stress levels, the temperature of the solid-solid transformation of the hydrocarbon component of a dental inlay wax was the determining factor of the resistance of the dental inlay wax to penetration. Annealed dental inlay waxes were more resistant to penetration than unannealed waxes.

\section{References}

1. Miller, G.W.: Total Thermal AnalysisPolymers as a Case in Point, Mat Res Stad 12: 8-21, 1972.

2. Craig, R.G.; Powers, J.M., and Peyton, F.A.: Differential Thermal Analysis of Commercial and Dental Waxes, J Dent Res 46: 1090-1097, 1967.

3. Midas-Mean Test, Ann Arbor, Mich: Statistical Research Laboratory, University of Michigan, 1972.

4. Edwards, R.T.: Crystal Habit of Paraffin Wax, Ind Eng Chem 49: 750-757, 1957.

5. HatT, H.H., and Lamberton, J.A.: Molecular Structure of Waxes, Res Sci Appl Ind 9: 138$146,1956$. 